\title{
Portal Hypertension as a Complication of Cystic Echinococcosis: A 20-Year Cohort Analysis
}

\begin{abstract}
Javier Collado Aliaga, ${ }^{1}$ Ángela Romero-Alegría, ${ }^{2}$ Montserrat Alonso-Sardón, ${ }^{3}$ Vanessa Prieto-Vicente, ${ }^{4}$ Amparo López-Bernus, ${ }^{5}$ Virginia Velasco-Tirado, ${ }^{6}$ Celia Sendra de la Ossa, ${ }^{7}$ Javier Pardo-Lledias, ${ }^{8}$ and Moncef Belhassen-García ${ }^{\star}$

${ }^{1}$ Servicio de Medicina Interna, Complejo Asistencial Universitario de Salamanca, Salamanca, Spain; ${ }^{2}$ Servicio de Medicina Interna, Complejo Asistencial Universitario de Salamanca, Instituto de Investigación Biomédica de Salamanca, Centro de Investigación de Enfermedades Tropicales de la Universidad de Salamanca, Universidad de Salamanca, Salamanca, Spain; ${ }^{3}$ Área de Medicina Preventiva y Salud Pública, Instituto de Investigación Biomédica de Salamanca, Centro de Investigación de Enfermedades Tropicales de la Universidad de Salamanca, Universidad de Salamanca, Salamanca, Spain; ${ }^{4}$ Servicio de Digestivo, Complejo Asistencial Universitario de Salamanca, Instituto de Investigación Biomédica de Salamanca, Salamanca, Spain; ${ }^{5}$ Servicio de Medicina Interna, Sección de Enfermedades Infecciosas, Complejo Asistencial Universitario de Salamanca, Instituto de Investigación Biomédica de Salamanca, Centro de Investigación de Enfermedades Tropicales de la Universidad de Salamanca, Salamanca, Spain;

${ }^{6}$ Servicio de Dermatología, Complejo Asistencial Universitario de Salamanca, Instituto de Investigación Biomédica de Salamanca, Centro de Investigación de Enfermedades Tropicales de la Universidad de Salamanca, Salamanca, Spain; 7 Sección de Enfermedades Infecciosas, Complejo Asistencial Universitario de Salamanca, Salamanca, Spain; ${ }^{8}$ Servicio de Medicina Interna, Hospital Marqués de Valdecilla Instituto de Investigación Marqués de Valdecilla, Avenida Valdecilla SN, 39008 Santander, Spain; ${ }^{9}$ Servicio de Medicina Interna, Sección de Enfermedades Infecciosas, Complejo Asistencial Universitario de Salamanca, Instituto de investigación Biomédica de Salamanca, Centro de Investigación de Enfermedades Tropicales de la Universidad de Salamanca, Universidad de Salamanca, Paseo San Vicente 58-182, 37007, Salamanca, Spain
\end{abstract}

\begin{abstract}
Cystic echinococcosis (CE) is a parasitic disease caused by the larval forms of species of the tapeworm Echinococcus. The most common location is the liver. To assess the frequency and clinical characteristics of portal hypertension $(\mathrm{PH})$ and the risk factors for $\mathrm{PH}$ development, we performed a retrospective observational study of inpatients diagnosed with hepatic CE and PH from January 1998 to December 2018, at Complejo Asistencial Universitario de Salamanca, Spain. Of 362 patients analyzed with hepatic CE, 15 inpatients (4.1\%) had a portal vein diameter $\geq 14 \mathrm{~mm}$, and the mean diameter of the portal vein was 16.9 (standard deviation [SD] \pm 2.1 ) $\mathrm{mm}$. Twelve patients were men. The mean age was 59.5 years $(\mathrm{SD} \pm$ 17.8 years). Four patients had ascites (26.6\%), four had collateral circulation (26.6\%), 14 had hepatosplenomegaly (93.3\%), five had esophageal varices (33.3\%), four had hematemesis, and three had jaundice. Other causes of PH included hepatitis $B$ virus (1 patient) and hepatitis $C$ virus (1 patient) infections and alcohol abuse (1 patient). The host variables associated with $\mathrm{PH}$ development were male sex (odds ratio, 4.6; 95\% confidence interval, 1.1-20.9; $P=0.030$ ) and larger cyst size (10.8 \pm 6.3 versus $7.6 \pm 4.1 ; P=0.004)$. Hepatic $C E$ is an infrequent cause of $P H$ that usually occurs without indications of liver failure. Larger cyst size and male sex were the main risk factors associated with this complication. Mortality was higher for patients with hepatic CE with $\mathrm{PH}$ than for patients with hepatic CE without $\mathrm{PH}$.
\end{abstract}

\section{INTRODUCTION}

Cystic echinococcosis (CE) is a parasitic disease with worldwide distribution caused by the larval forms of species of the tapeworm Echinococcus granulosus sensu lato; dogs are a definitive host and humans are an accidental host. ${ }^{1}$ Human echinococcosis is prevalent in northern and eastern Africa, central Asia, South America, Australia, and Mediterranean Basin countries, such as Greece, Italy, Portugal, and Spain. $^{2}$

Human infection causes cysts in any organ, although the most common locations are the liver (>65\%) and lung $(25 \%) .{ }^{3}$ CE can be acquired at any time, with the risk increasing with increased time exposed to or living in endemic areas. Infected patients are frequently asymptomatic for years or decades. ${ }^{4}$ Accidental findings of CE are sometimes found during clinical autopsies. ${ }^{5}$

Complications of CE or CE recurrence can occur secondary to surgical procedures ${ }^{6,7}$ because of the need to handle the cyst, spontaneous rupture of the cyst wall with the development of a fistula (biliary, bronchial, pleural, and other types), and superinfection by bacterial or fungal species. ${ }^{8}$

\footnotetext{
*Address correspondence to Moncef Belhassen-García, Servicio de Medicina Interna. Sección de Enfermedades Infecciosas, Complejo Asistencial Universitario de Salamanca, Instituto de Investigación Biomédica de Salamanca, Centro de Investigación de Enfermedades Tropicales de la Universidad de Salamanca, Universidad de Salamanca, Paseo San Vicente 58-182, 37007, Salamanca, Spain. E-mail: mbelhassen@hotmail.com
}

Additionally, immunological reactions such as urticaria and anaphylaxis have been observed with complications caused by cysts.

During a recent retrospective study, we evaluated spontaneous complications of CE. Unexpectedly, we found that some patients with hepatic CE presented clinical characteristics of portal hypertension $(\mathrm{PH})$, such as ascites and variceal hemorrhage. ${ }^{9}$ Therefore, in the present study, we aimed to examine the frequency and clinical characteristics of $\mathrm{PH}$ and the risk factors for $\mathrm{PH}$ development in patients with hepatic CE.

\section{PATIENTS AND METHODS}

Study design and participant selection. A retrospective, longitudinal, descriptive study was designed to review all patients diagnosed with CE at Complejo Asistencial Universitario de Salamanca (CAUSA) between January 1998 and December 2018. CAUSA is a tertiary care hospital that covers an area of $12350 \mathrm{~km}^{2}$. In, 2018, there were 333,603 inhabitants (National Institute of Statistics [INE\}; http://www.ine. es/) located in midwestern Spain.

All patients included in the study fulfilled the following four criteria: diagnosed with CE according to code 122 of the International Classification of Disease, 9th edition, Clinical Modification (ICD-9-CM) during 1998 to 2015, or with code B67 of the International Classification of Disease, 10th edition (ICD10) during 2016 to 2018; liver disease caused by Echinococcus granulosus; highly suggestive clinical setting of $\mathrm{PH}$, such as encephalopathy, splenomegaly, esophageal varices, variceal hemorrhage, portal hypertensive gastropathy, and 
ascites; and radiological signs consistent with $\mathrm{PH}$ using ultrasonography/tomography or higher than F3 using FibroScan (Echosens, Waltham, MA). The exclusion criteria were missing data, extrahepatic $C E$, and no adequate portal image. The clinical and epidemiological data were collected after a review of the medical records. All data analyzed were anonymized.

We defined $\mathrm{PH}$ syndrome if the patient had symptoms or signs usually associated with $\mathrm{PH}$ (including variceal hemorrhage, portal hypertensive gastropathy, ascites, and spontaneous bacterial peritonitis) and radiological signs on ultrasonography or tomography (such as ascites, splenomegaly, portal flow mean velocity $<12 \mathrm{~cm} /$ second, inversion of flow in the portal vein, portosystemic collaterals of a patent paraumbilical vein or spleen-renal collaterals, dilated left and short gastric veins, portal vein diameter $>13 \mathrm{~mm}$, decreased or no respiratory variation in the diameter of the splenic and superior mesenteric veins, and portal, splenic, or superior mesenteric vein thrombosis). We also considered $\mathrm{PH}$ if liver stiffness was $>13.6 \mathrm{kPa}$ as measured by FibroScan (FibroScan Touch 502; Echosens).

Data analysis. To perform statistical analyses, descriptive analysis was conducted for each individual variable. For categorical variables, the results are expressed as absolute values $(n)$, proportions $(n / N)$, or percentages (\%). For quantitative variables, the results are expressed as the mean and standard deviation (SD), median and interquartile range (IQR; quartile 1-quartile 3 ), and range (minimum value-maximum value). The strength of the association between categorical variables was measured using Pearson's $\chi^{2}$ contrast statistic and odds ratio (OR) estimates. An analysis of variance (F-test) was used to statistically assess the equality of means between groups. $P$ $\leq 0.05$ was considered statistically significant (significance level of 0.05). The SPSS 26.0 (SPSS, IBM, Armonk, NY) statistical package was used.

Ethics statement. The study protocol was approved by the Clinical Research Ethics Committee of the Complejo Asistencial Universitario de Salamanca (Salamanca, Spain). The procedures described here were performed in accordance with the ethical standards described in the 2013 revised Declaration of Helsinki.

\section{RESULTS}

Between January 1998 and December 2018, a total of 594 patients received a diagnosis of CE; 88 patients with no data were excluded and 506 inpatients with a diagnosis of $\mathrm{CE}$ were included in the initial study. After checking all cases, 144 patients were excluded because of extrahepatic CE without hepatic involvement (65), the absence of an adequate portal image, or nonvalid imaging test results (79). Therefore, 362 $(71.5 \%)$ patients underwent portal studies via radiological examinations (ultrasonography, computed tomography, or magnetic resonance imaging) or elastography, including complementary tests, and they were included as study subjects. Fifteen (4.1\%) included patients fulfilled the criteria for PH syndrome. Figure 1 shows the flowchart of participant inclusion in this study.

Clinical and epidemiological data of the 15 patients with CE and $\mathrm{PH}$ are shown in Table 1. Twelve patients (80\%) were male and three (20\%) were female. The mean age ( \pm SD) of the sample was 59.5 years ( \pm 17.8 years) (median, 61 years; IQR, 50-70 years; range, 22 to 93 years). These patients presented with one hepatic cyst (10 patients; $66.6 \%$ ), two cysts (4 patients; $26.6 \%$ ), and three or more (1 patient; 6.6\%). Cysts were most frequently located in liver segments VII and VIII (5 patients; 33.3\%) (Figure 2) The World Health Organization (WHO) classifications most frequently presented were stage II (7 patients; 46.7\%), stage V (4 patients; $26.7 \%)$, stage IV (2 patients; 13.3\%), and stage III (1 patient; 6.7\%). For one patient, there were no detailed radiological data. The mean size ( $\pm S D$ ) of the cysts was $10.7 \mathrm{~cm}(S D \pm 6.3) \mathrm{cm}$ (median, $10 \mathrm{~cm}$; IQR 5-16; range, 3-23 cm). For 9 (60\%) patients, the mean cyst size was larger than $7 \mathrm{~cm}$.

We studied the risk factors for $\mathrm{PH}$ in patients with hepatic CE. Among the variables of the host associated with $\mathrm{PH}$, only male sex was associated an increased risk of $\mathrm{PH}(\mathrm{OR}$,

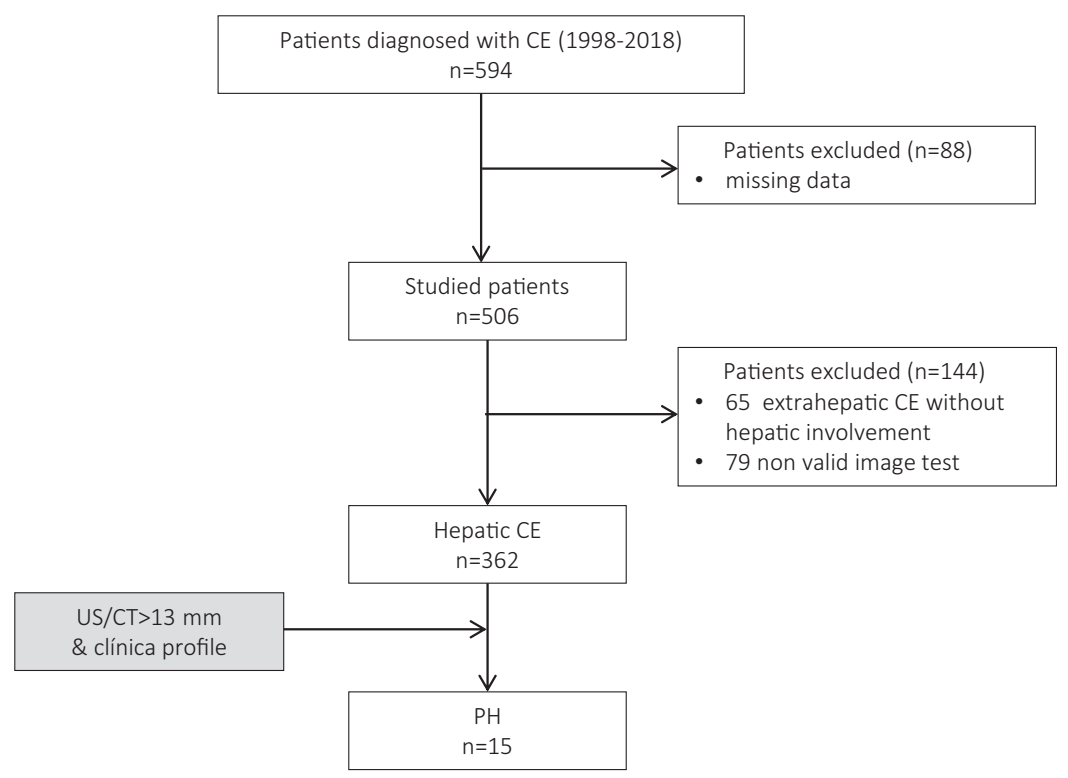

Figure 1. Participant profile. 
COLLADO ALIAGA AND OTHERS

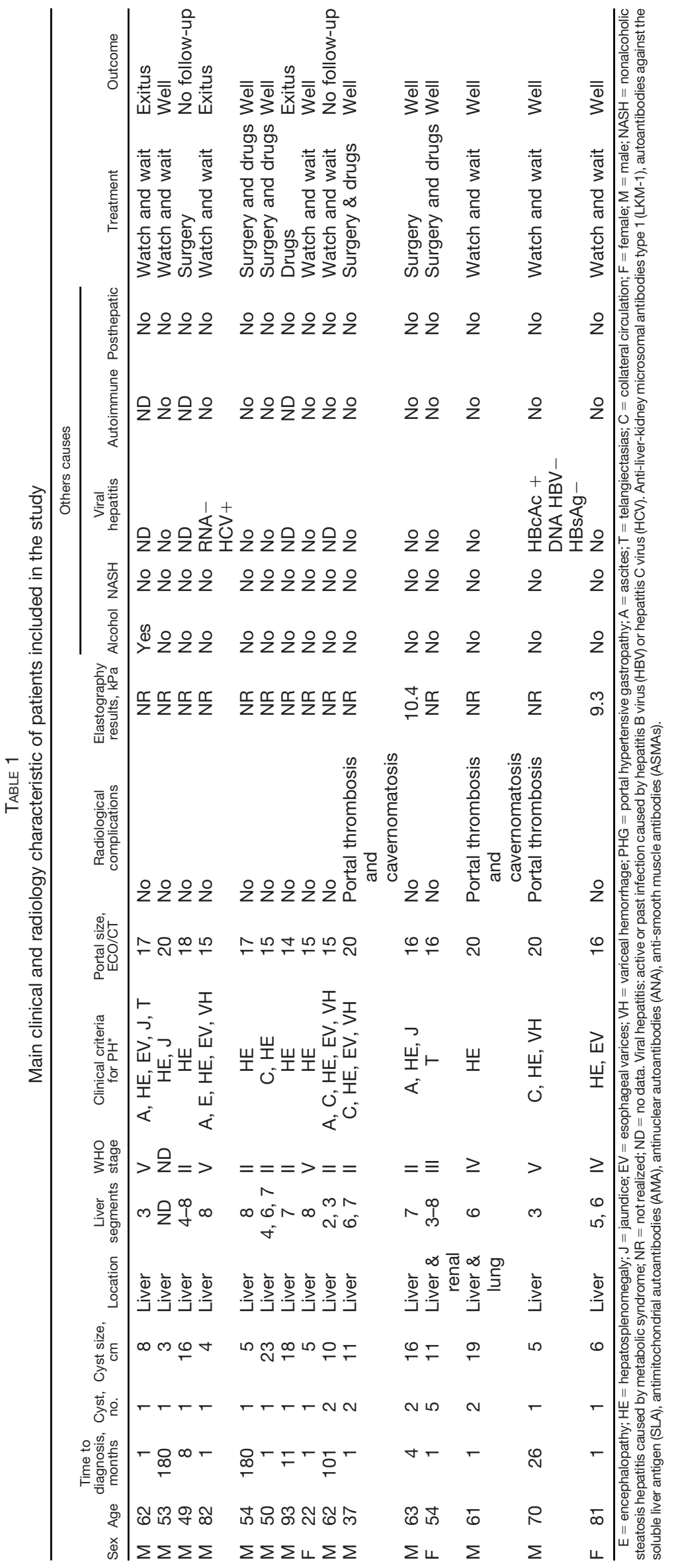




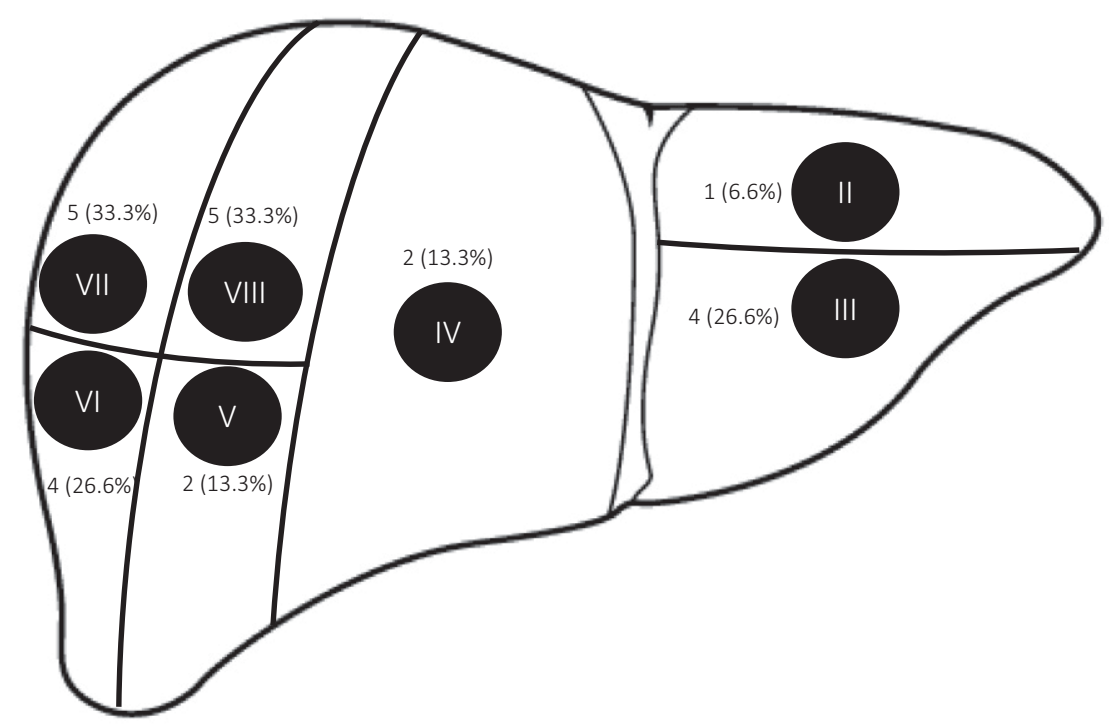

FIGURE 2. Main cyst location for patients with cystic echinococcosis included in the study.

4.6; 95\% Cl, 1.1-20.9; $P=0.030$ ). Patients with hepatic CE with $\mathrm{PH}$ had larger cysts than patients with hepatic $\mathrm{CE}$ without $\mathrm{PH}(10.8 \pm 6.3$ compared with $7.6 \pm 4.1 ; P=0.004)$.

The mean diameter of the portal vein was $16.9 \mathrm{~mm}$ (SD \pm 2.1 $\mathrm{mm}$ ) (median, $16 \mathrm{~mm}$; IQR, 15-20; range, 14-20 mm). The signs most frequently found during radiological examination were portal vein diameter $>14 \mathrm{~mm}$ (15 patients; $100 \%$ ), ascites (4 patients; $12 \%$ ), splenomegaly (7 patients; $21 \%$ ), portosystemic collaterals ( 5 patients; $15 \%$ ), portal thrombosis (2 patients; $13.3 \%$ ), and cavernomatosis (1 patient; 6.6\%). Two patients had elastography results of $9.85(\mathrm{SD} \pm 0.55) \mathrm{kPa}$.

The most frequently observed symptoms or signs associated with $\mathrm{PH}$ were hepatosplenomegaly (2 patients; $14.3 \%$ ), ascites (4 patients; $26.6 \%$ ), esophageal varices (5 patients; $33.3 \%$ ), hematemesis (4 patients; $26.6 \%$ ), and jaundice (3 patients; $20 \%)$. There were asymptomatic patients with cytopenia (thrombocytopenia, leukopenia, and anemia) and hypoalbuminemia. Among the studied patients, other causes of $\mathrm{PH}$ were detected in 3 (20\%) patients: alcohol abuse for 1 (6.6\%) patient, hepatitis $B$ virus infection for 1 patient, and hepatitis $C$ virus infection for 1 patient. The patients analyzed did not have liver failure, and their mean Child-Pugh score was 5.86 (SD \pm 1.08). Other posthepatic causes of $\mathrm{PH}$ such as suprahepatic vein or cava vein thrombosis were ruled out with ultrasonography and tomography studies. No patient had right heart failure.

During a mean follow-up of 70.9 (SD \pm 64.5 months), 10 (66.6\%) patients had positive evolution, 2 (13.3\%) patients had no follow-up data available, and 1 male patient died because of $\mathrm{PH}$ complications. Mortality was higher for patients with hepatic CE with $\mathrm{PH}$ than for patients with hepatic $\mathrm{CE}$ without $\mathrm{PH}$, although the difference was not significant.

\section{DISCUSSION}

$\mathrm{PH}$ syndrome has often been described as a complication of different parasitic diseases. The most frequent parasitic infection associated with $\mathrm{PH}$ is schistosomiasis, which is caused by chronic infection of a trematode species of Schistosoma such as S. mansoni or S. japonicum. ${ }^{10}$ The authors found that more than $5 \%$ of all patients with intestinal schistosomiasis had a severe form of hepatosplenic schistosomiasis and, consequently, $\mathrm{PH} .{ }^{11}$ However, another species of Echinococcus, Echinococcus multilocularis, has also been established as a cause of $\mathrm{PH}$ because of its characteristic invasion of liver tissue. $^{12}$

Unlike previous parasites, CE has never been included (to our knowledge) on the list of causes of $\mathrm{PH}$. However, this syndrome has been referred to in case records in the literature. ${ }^{13,14}$ Therefore, we aimed to assess the frequency, risk factors, clinical characteristics, and prognosis of $\mathrm{PH}$ in our cohort of patients with hepatic CE.

An academic definition of $\mathrm{PH}$ is portal vein pressure higher than $5 \mathrm{mmHg} .{ }^{15}$ Nevertheless, to measure this pressure, it is necessary to introduce a venous catheter into the suprahepatic veins. However, this technique for the diagnosis of $\mathrm{PH}$ syndrome is not usually performed outside of specialized hemodynamic and transplantation liver units. In clinical practice, we usually diagnose $\mathrm{PH}$ syndrome if patients have liver or systemic vein portal disease in addition to clinical characteristics of secondary complications of $\mathrm{PH}$, such as ascites, splenomegaly, and variceal hemorrhage, or suggestive radiological signs.

Therefore, during this retrospective study, we included only patients who presented unequivocal symptoms or signs associated with $\mathrm{PH}$ and complementary signs on radiological (ultrasonography, computed tomography, or magnetic resonance imaging) or elastography examinations. Computed tomography and magnetic resonance imaging do not offer functional imaging to establish a diagnosis of $\mathrm{PH}$; however, they detect secondary changes in $\mathrm{PH}$, such as portosystemic collateral formation, splenomegaly, portal hypertensive gastropathy, and ascites. ${ }^{16}$

During our study, we found 15 patients with clinical and complementary data suggestive of $\mathrm{PH} ; 4 \%$ of those patients had hepatic $\mathrm{CE}$. This low frequency of $\mathrm{PH}$ syndrome supports the scarcity of data presented in the literature. Therefore, it is 
possible that those patients with long-term parasitic infection have a higher possibility of $\mathrm{PH}$ development. However, during our study, we did not find an association between older age and $\mathrm{PH}$ or between comorbidities and $\mathrm{PH}$. Our data showed no association of $\mathrm{PH}$ with the stage or location of the cyst inside the liver; however, it did show an association with the cyst size. Moreover, there is no clear relationship between $\mathrm{PH}$ and endovascular invasion of the parasite because only three patients had portal thrombosis.

The patterns of symptoms and signs detected in patients with $\mathrm{PH}$ and $\mathrm{CE}$ suggested differences between schistosomiasis and E. multilocularis infection. Schistosomiasis usually causes presinusoidal $\mathrm{PH}$, and patients have ascites, hepatosplenomegaly, and variceal hemorrhage. ${ }^{17}$ Alveolar echinococcosis spreads to suprahepatic veins, and the pattern is usually postsinusoidal $\mathrm{PH}$ with clinical characteristics compatible with Bud-Chiari syndrome and indications of liver failure. In a series from Poland, Echinococcus multilocularis was a cause of transplantation for $13 \%$ of patients. ${ }^{18}$

Patients with $\mathrm{PH}$ associated with $\mathrm{CE}$ usually present with abdominal pain, ascites, splenomegaly, variceal hemorrhage, and encephalopathy, but no indications of liver failure. However, it was an accidental finding for approximately one-third patients. $^{19}$

We know that $\mathrm{PH}$ is a cause of mortality for patients with schistosomiasis or E. multilocularis infection. In our cohort, we found a mortality rate of $20 \%$ for patients with hepatic CE who had $\mathrm{PH}$, which was higher than the rate we found for our overall cohort. We previously reported that, for patients with $\mathrm{CE}, \mathrm{PH}$ was the main cause of death. Superinfections and biliary fistula were the main causes of mortality in that cohort. During this study, we found that $\mathrm{PH}$ complications could also be causes of mortality.

The pathogenic mechanisms involved in the development of $\mathrm{PH}$ in patients with $\mathrm{CE}$ are not clear. It is possible that the presence of a cyst could increase the resistance of portal blood flow into the liver. This possibility is consistent with the larger cyst size observed in patients with $\mathrm{CE}$ with $\mathrm{PH}$ than that in patients with $\mathrm{CE}$ without $\mathrm{PH}$. These data support the idea that liver tissue compression caused by cysts could explain $\mathrm{PH}$. However, we did not find an association between the cyst location and $\mathrm{PH}$; therefore, direct compression of the portal vein is unlikely to be the mechanism of $\mathrm{PH}$ development involved in this cohort.

There could be factors related to endothelial dysfunction in patients with CE, however. Nitric oxide (NO) is a molecule associated with vasodilator effects in the portal vein. Therefore, NO donor drugs, such as nitrates, are frequently used in the treatment of $\mathrm{PH}$ syndrome. Some parasitic infections, such as Schistosoma mansoni, have been found to alter endothelial cell function, thus decreasing the expression of endothelial NO synthase and, consequently, NO production. ${ }^{20}$ Patients with CE have NO concentrations lower than those of the healthy population and present a slight increase after surgery. ${ }^{21}$

Finally, liver fibrosis induced by CE has been observed in an experimental mouse model of infection. ${ }^{22}$ In our cohort, it was interesting that liver fibrosis was demonstrated in two of the only two patients who underwent liver elastography (F3 stage of fibrosis) using FibroScan (FibroScan Touch 502; Echosens).

Various mediators are involved in liver fibrosis. It has been proposed that vascular endothelial growth factor acts in the
$\mathrm{PH}$ development pathway, and that its inhibition improves $\mathrm{PH}$, hyperdynamic splanchnic circulation, portosystemic collateralization, and liver fibrosis. ${ }^{23}$ Egg antigens of Schistosoma and other parasitic infections cause VEGF upregulation in endothelial cells. ${ }^{24}$ E. granulosus has also been shown to increase VEGF in infected patients. ${ }^{21}$ VEGF is associated with fibrogenesis periportal fibrosis and angiogenesis, and it could be involved in the PH development pathway. ${ }^{25}$

Another mediator involved in fibrosis in patients with CE is transforming growth factor- $\beta$. E. granulosus increases the level of transforming growth factor- $\beta 1$ in advanced stages or infection, thus contributing to the development of the fibrocyst wall and inhibiting the expression of miR-19, consequently promoting liver fibrosis and secondary $\mathrm{PH}^{26}$

Finally, in our cohort of patients with hepatic $\mathrm{CE}$ and $\mathrm{PH}$, we found other possible causes of $\mathrm{PH}$, such as alcohol abuse and hepatitis $C$ and $B$ virus infections, in only three patients. Although it is difficult, especially during retrospective studies, to assess how different factors can contribute to $\mathrm{PH}$, we think that $\mathrm{CE}$ could have had an important role in the development of $\mathrm{PH}$ in these patients. This fact has been highlighted for other parasitic infections as well; therefore, hepatosplenic schistosomiasis patients coinfected with HCV and HBV could be at higher risk for $\mathrm{PH}^{27}$

Based on all these findings, it is difficult to know the definitive cause of $\mathrm{PH}$. It is possible that it could be caused by multiple factors, such as portal compression, invasion of the portal system, and induction into fibrosis.

Our work was retrospective; therefore, it had some limitations, such as a scarce number of patients and limited selection criteria. Nevertheless, it is difficult to perform other types of studies of CE because of the chronic characteristics of this infection and its long follow-up. Therefore, the results should be interpreted with caution. Multicenter works should be performed in the future to verify our results.

\section{CONCLUSIONS}

Liver $\mathrm{CE}$ is a rare cause of $\mathrm{PH}$, which usually occurs without indications of liver failure. Larger cyst size and male sex were the main risk factors associated with this complication of $\mathrm{CE}$. Mortality was higher for patients with hepatic $\mathrm{CE}$ with $\mathrm{PH}$ than for patients with hepatic CE without $\mathrm{PH}$.

Received March 4, 2021. Accepted for publication April 28, 2021.

Published online July 19, 2021.

Authors' addresses: Javier Collado Aliaga, Servicio de Medicina Interna, Complejo Asistencial Universitario de Salamanca, Salamanca, Spain, E-mail: humanoide123@hotmail.com. Angela Romero-Alegría, Servicio de Medicina Interna, Complejo Asistencial Universitario de Salamanca, Instituto de Investigación Biomédica de Salamanca, Centro de Investigación de Enfermedades Tropicales de la Universidad de Salamanca, Universidad de Salamanca, Salamanca, Spain, E-mail: aralegria82@hotmail.com. Montserrat Alonso-Sardón, Área de Medicina Preventiva y Salud Pública, Instituto de Investigación Biomédica de Salamanca, Centro de Investigación de Enfermedades Tropicales de la Universidad de Salamanca, Universidad de Salamanca, Salamanca, Spain, E-mail: sardonm@usal.es. Vanessa Prieto-Vicente, Servicio de Digestivo, Complejo Asistencial Universitario de Salamanca, Instituto de Investigación Biomédica de Salamanca, Salamanca, Spain, E-mail: vanessapv_78@hotmail.com. Amparo López-Bernus, Servicio de Medicina Interna, Sección de Enfermedades Infecciosas, Complejo Asistencial Universitario de Salamanca, Instituto de Investigación Biomédica de Salamanca, Centro de Investigación de Enfermedades Tropicales de la Universidad de Salamanca, Salamanca, 
Spain, E-mail: albernus@hotmail.com. Virginia Velasco-Tirado, Servicio de Dermatología, Complejo Asistencial Universitario de Salamanca, Instituto de Investigación Biomédica de Salamanca, Centro de Investigación de Enfermedades Tropicales de la Universidad de Salamanca, Salamanca, Spain, E-mail: virvela@yahoo.es. Celia Sendra de la Ossa, Sección de Enfermedades Infecciosas, Complejo Asistencial Universitario de Salamanca, Salamanca, Spain, E-mail: celiasendra@gmail.com. Javier Pardo-Lledias, Servicio de Medicina Interna, Hospital Marqués de Valdecilla Instituto de Investigación Marqués de Valdecilla, Avenida Valdecilla SN, 39008 Santander, Spain, E-mail: javipard2@hotmail.com. Moncef Belhassen-García, Servicio de Medicina Interna, Sección de Enfermedades Infecciosas, Complejo Asistencial Universitario de Salamanca, Instituto de Investigación Biomédica de Salamanca, Centro de Investigación de Enfermedades Tropicales de la Universidad de Salamanca, Universidad de Salamanca, Paseo San Vicente 58-182, 37007, Salamanca, Spain, E-mail: mbelhassen@hotmail.com.

\section{REFERENCES}

1. Thompson RCA, McManus DP, 2002. Towards a taxonomic revision of the genus Echinococcus. Trends in Parasitology 18: 452-457.

2. Jenkins DJ, Romig T, Thompson RCA, 2005. Emergence/reemergence of Echinococcus spp.-a global update. Int J Parasitol 35: 1205-1219.

3. Pedrosa I, Saíz A, Arrazola J, Ferreirós J, Pedrosa CS, 2000. Hydatid disease: radiologic and pathologic features and complications. Radiographics 20: 795-817.

4. Armiñanzas C, Gutiérrez-Cuadra M, Fariñas MC, 2015. Hydatidosis: epidemiological, clinical, diagnostic and therapeutic aspects. Revista Espanola de Quimioterapia: Publicacion Oficial de la Sociedad Espanola de Quimioterapia 28: 116-124.

5. Schenone $\mathrm{H}, 1989$. Cysticercosis and hydatidosis do not always bring about detectable pathology in humans. Boletín Chileno de Parasitología 44: 63-65.

6. Velasco-Tirado V et al., 2017. Recurrence of cystic echinococcosis in an endemic area: a retrospective study. BMC Infect Dis 17: 455.

7. Velasco-Tirado V et al., 2018. Management of cystic echinococcosis in the last two decades: what have we learned? T Roy Soc Trop Med H 112: 207-215.

8. García MB, Lledías JP, Pérez IG, Tirado VV, Pardo LF, Bellvís LM, Varela G, Sánchez MC, 2010. Primary super-infection of hydatid cyst-clinical setting and microbiology in 37 cases. Am J Tropical Medicine Hyg 82: 376-378.

9. Collado-Aliaga J, Romero-Alegría Á, Alonso-Sardón M, Muro A, López-Bernus A, Velasco-Tirado V, Bellido JLM, Pardo-Lledias J, Belhassen-García M, 2019. Complications associated with initial clinical presentation of cystic echinococcosis: a 20-year cohort analysis. Am J Trop Med Hyg 101: 628-635.

10. Silva LCD, Carrilho FJ, 1992. Hepatosplenic schistosomiasis. Pathophysiology and treatment. Gastroenterol Clin N 21: 163177.

11. Strauss E, 2002. Hepatosplenic schistosomiasis: a model for the study of portal hypertension. Ann Hepatol 1: 6-11.
12. Sezgin O, Altintaș E, Saritaș U, Sahin B, 2005. Hepatic alveolar echinococcosis: clinical and radiologic features and endoscopic management. J Clin Gastroenterol 39: 160-167.

13. García-Díaz JD, Ramos JCR, 2001. Portal hypertension as complication of hepatic hydatidosis. Anales De Medicina Interna Madrid Spain 1984 18: 608-609.

14. López CSJ, Sáez ML, Delgado AB, Martín MC, Toutouh SH, Sáenz JLV, 2018. Portal hypertension as a complication of hepatic hydatidosis. Gastroenterol Hepatol. doi: 10.1016/ j.gastrohep.2018.01.002.

15. Lee CK, 2017. Biomarkers and imaging studies to predict portal hypertension and varices. Clin Liver Dis 9: 94-98.

16. Leung JC-F, Loong TC-W, Pang J, Wei JL, Wong VW-S, 2018. Invasive and non-invasive assessment of portal hypertension. Hepatol Int 12: 44-55.

17. Junior RLK, 2013. Comment on: relationship between splenomegaly and hematologic findings in patients with hepatosplenic schistosomiasis. Rev Bras Hematol Hemoter 35: 378-379.

18. Sulima $\mathrm{M}$ et al., 2016. Liver transplantation for incurable alveolar echinococcosis: an analysis of patients hospitalized in Department of Tropical and Parasitic Diseases in Gdynia. Transplant Proc 48: 1708-1712.

19. Romig T, Zeyhle E, Macpherson CN, Rees PH, Were JB, 1986. Cyst growth and spontaneous cure in hydatid disease. Lancet 1: 861.

20. Oliveira SDS, Quintas LEM, Amaral LS, Noël F, Farsky SH, Silva CLM, 2011. Increased endothelial cell-leukocyte interaction in murine schistosomiasis: possible priming of endothelial cells by the disease. PLoS One 6: e23547.

21. Matera $G$ et al., 2018. Increase of vascular endothelial growth factor and decrease of mcp-1 and some updated epidemiology aspects of cystic echinococcosis human cases in Calabria region. Mediators Inflamm 2018: 1-11.

22. Liedtke C, Luedde T, Sauerbruch T, Scholten D, Streetz K, Tacke F, Tolba R, Trautwein C, Trebicka J, Weiskirchen R, 2013. Experimental liver fibrosis research: update on animal models, legal issues and translational aspects. Fibrogenesis Tissue Repair 6: 19.

23. Gana JC, Serrano CA, Ling SC, 2016. Angiogenesis and portalsystemic collaterals in portal hypertension. Ann Hepatol 15: 303-313.

24. Loeffler DA, Lundy SK, Singh KP, Gerard HC, Hudson AP, Boros DL, 2002. Soluble egg antigens from Schistosoma mansoni induce angiogenesis-related processes by up-regulating vascular endothelial growth factor in human endothelial cells. $J$ Infect Dis 185: 1650-1656.

25. Shariati F, Pérez-Arellano JL, Carranza C, López-Abán J, Vicente B, Arefi M, Muro A, 2011. Evaluation of the role of angiogenic factors in the pathogenesis of schistosomiasis. Exp Parasitol 128: 44-49.

26. Niu F, Chong S, Qin M, Li S, Wei R, Zhao Y, 2019. Mechanism of fibrosis induced by Echinococcus spp. Dis 7: 51.

27. Gad A, Tanaka E, Orii K, Rokuhara A, Nooman Z, Serwah AH, Shoair M, Yoshizawa K, Kiyosawa K, 2001. Relationship between hepatitis $C$ virus infection and schistosomal liver disease: not simply an additive effect. $J$ Gastroenterol 36: 753-758. 